\title{
Using computer-mediated conferencing to facilitate group projects in an educational psychology course
}

\author{
MARGARET D. ANDERSON \\ State University of New York College at Cortland, Cortland, New York
}

\begin{abstract}
A course design is presented as a model for incorporating computer-mediated conferencing (CMC) in support of administering, organizing, and evaluating group projects in a psychology course. Students in an educational psychology course use e-mail and an electronic bulletin board system to communicate within and between student groups as well as with the instructor. Critical elements for the implementation of CMC are discussed, and suggestions for ways to avoid problems are offered.
\end{abstract}

Various models for incorporating the use of computers to enhance communication in academic courses have been developed over the past 5 years (Mason, 1993). The use of computer-mediated communication (CMC) has enjoyed both success and failure (Albrektson, 1995), which are often attributable to the reasons for, and implementation of, the CMC component. Before attempting to employ a CMC course component, the instructor needs to be aware that students are being introduced not only to a new medium, but also to a new method of interacting with their peers and professor. Most students are not accustomed to committing their ideas to writing and sharing them with their (faceless) collaborators. This manner of interaction requires the development of new skills and abilities.

One reason that $\mathrm{CMC}$ components often fail is the same one that dooms many attempts at incorporating the use of computers in any format into courses: the instructor is fascinated with the technology and searches for a way to use it, regardless of the pedagogical soundness of the activity (Albrektson, 1995). The present paper reports on the use of a CMC component to support a pedagogically proven technique: the collaborative group project (Slavin, 1990). The group project in question constituted one of the learning activities in an educational psychology course offered at SUNY College at Cortland.

\section{Training in the Medium}

Since the students enrolled in the course have little prior experience of computers, it is necessary to introduce them to basic computer skills prior to commencing the project. During the 1st week of the semester, a networked computer laboratory facility on campus is reserved for one class period to introduce students to the basics of the e-mail system. Subsequently, students complete a graded home-

Correspondence concerning this article should be addressed to M. D. Anderson, Psychology Dept., SUNY College at Cortland, Cortland, NY 13045 (e-mail: andersmd@syncorva.bitnet). work assignment in which they must set their personal name in their return address; set the carbon copy prompt in their address (so that the instructor can receive a copy of their work on e-mail); write to the next person on the class distribution list (which has been sent to them electronically); and put the distribution list and the instructions for completing the first assignment into a mail folder. This first assignment is important not only to achieve certain administrative functions, but also to ensure that everyone gets back to one of the campus computer labs and logs on to e-mail to become a little more comfortable with the system.

During the 2nd week of the semester, the course meets in the computer lab for one additional class period. At that time, students are introduced to the use of the Vax Notes conferencing system. In addition, they are provided with an explanation of the differences between, and uses for, e-mail and conferencing systems. The etiquette relating to electronic communication is also discussed. Following this introduction, students join a private electronic conference created specifically for the administration of the group projects. Students pair up for both of these initial computer training sessions so that they may meet another class member and have someone with whom to struggle through these initial assignments.

It is essential to the project's success to ensure that all students in the course are familiar with the fundamentals of the specific systems they will be using, even if they are familiar with computers in general. It is most expedient to do this in a single training session for each system. In addition to the physical training sessions, the availability of detailed handouts on the use of both e-mail and the conferencing system is critical.

\section{Design of the Group Project}

Four-person groups are constructed by the instructor, and each group is assigned a topic and a primary research article. The first mechanical step in engaging in the group project requires each student to obtain his/her assignment from the electronic class conference. These assign- 
ments consist of the names of the four group members, the title of their assigned article, and the dates by which various components of the project must be completed. The second step in the process is for each student to introduce themselves to the other members of the group by using e-mail. These two tasks serve to reinforce the difference between the use of e-mail and of conferencing systems. It is important to ensure that students develop a sense of confidence in the medium, and these first, simple requirements usually guarantee that. In addition, the "introduction" assignment allows them to "meet" the members of their group and facilitates future communication.

As for the actual content of the presentation, students must first read their assigned article. Next they are each required to locate a related journal article. This allows them to practice on-line literature search techniques. As soon as each student has located an article, he/she uses e-mail to provide the group members with the reference and a brief description of the paper.

The next step of the project requires each student to write a summary of the article selected, and to post this summary, along with the complete reference, in the designated area for the student's group in the class electronic conference.

Once the abstracts have been posted to the conference, class members who are not part of the "active group" have a week to read the primary article in the text and the abstracts on the conferencing system. Each class member is then required to generate a question for the presenting group. These questions are entered into the group Topic on the electronic conference and are used by the group when preparing their oral presentation. Receiving the questions ahead of time allows presenters to research and prepare complete answers rather than respond to impromptu questions in class.

After the group has received all the class questions, they set about determining the format they wish to use to present their material. The organization of the oral presentation, the preparation of answers to the class questions, and final coordination of the project take place by e-mail. Some of the groups are much better at using the electronic medium for this sort of communication; others use e-mail primarily to determine when and where they can physically meet to work on the project. It is particularly interesting to monitor the progress of the groups (through copies of all of their e-mail communication) as they try to make physical contact and finally end up by saying "guess we may HAVE to do this electronically" because they cannot coordinate schedules. It does not take long for these groups to appreciate the flexibility afforded by the use of e-mail for creating virtually continuous work groups in which each can participate at a convenient time. From the instructor's point of view, the ability to monitor the groups in action makes it possible to respond to questions or calls for help; to make suggestions if the group seems to be floundering; and to assess the input of individual members.

The oral presentations are scheduled during regular class sessions and must be at least $20 \mathrm{~min}$ and no more than $30 \mathrm{~min}$ in length. There are no restrictions on presentation format; the general guidelines are that each member of the group must take an active part, must teach the class the material he/she has been assigned, and must try to make the presentations as interesting as possible. Students have been very creative in designing their presentations, sometimes using game show formats, debates, or question-and-answer sessions. Some groups have conducted surveys in class to illustrate their topics; others have incorporated video clips or slides. Some groups have resorted to more traditional lecture presentations, but generally report later that they were unhappy with this format and do not repeat it for their second project.

\section{Project Grades}

The group project is assessed in five equally weighted components. Three components are based on individual performance: quality of the abstract; participation in organizing the presentation; and quality of the student's component of the oral presentation. One component is dependent on the entire group: overall group presentation quality. The final component is determined by peer assessment (completed by class members) for the presenting individuals and the entire group. Grading the project in this manner emphasizes the importance of the various elements of the overall project, as well as identifies aspects of individual and group accountability. Such detailed assessment allows differential grading based on individual contributions, which can be accurately gauged only by continuous access to the students' activity on e-mail and the electronic conferencing system.

\section{Tips for Successful Implementation of a CMC Component}

Certain elements need to be attended to in order to ensure that a CMC component will succeed. (1) One must emphasize the importance of computer literacy skills as a prerequisite to academic success. Students need to understand that they are not taking a computer course, but are being exposed to a new way of conducting academic projects, and that computer skills are similar to reading and writing requirements in a college course. (2) One must conduct a thorough introduction to e-mail and the electronic conferencing system to ensure that all students are competent with those systems. This includes providing written handouts that can be referred to later. (3) One should be very specific about the directions for completing various components of the project; setting explicit deadlines for completion of the various components also helps to reduce procrastination and avert problems caused by fear of using the medium. (4) One should have students do at least two projects with the same format. During the first project, most students are struggling to grasp the basic mechanics of the assignment and computer systems. By the second project, they realize how easy the mechanical aspects are and they are able to concentrate on the content and the quality of the oral presentations. (5) One should allow much more time to administer these projects than one would ever estimate. Students need help in using 
the media, locating articles, communicating with their groups, and preparing their presentations. The faculty member needs time to generate the handouts, to monitor the activity of the groups, to create the project structure on the conferencing system, to act as moderator for the conference, to change entry titles for abstracts or questions, and to correct locations and message numbers when necessary. (6) The technology, or the project systems will fail at some time during the semester. This is an excellent opportunity to teach students (and faculty) problem-solving skills, flexibility, and patience.

\section{Conclusions}

While this format is certainly not without its problems, coupling the unique advantages of the electronic environment with the traditional benefits of group projects enhances student interactivity, as well as improves research, writing, and oral presentation skills. Most students acknowledge that while they are hesitant (or actually terrified) to learn to use a computer, it is a skill they will need in the job market and even to survive in the world of the future. Once students learn how to use the system and continue to do so throughout the semester, most lose their reluctance to work on the computers and begin to ask what else they can do on the network and try to locate other courses in which computers are used. Students also come to appreciate the flexibility afforded by computer conferencing in collaborative projects.

Using electronic media to facilitate group projects retains the advantages of traditional group projects, increas- ing class interaction, and improving oral presentation, research, and writing skills. At the same time incorporating electronic communication has a number of unique advantages: (1) Getting group members together outside of class, always a problem given varying student schedules, becomes almost impossible with the increasing enrollment of "nontraditional students." By "meeting" electronically, groups can communicate without space or time restrictions, creating potentially continuous dialogues. (2) Coordinating the groups electronically allows the professor to sit in on these dialogues. He/she can be an unobtrusive observer, instantly available to answer questions, or give advice if the group seems to need it. (3) Monitoring the groups at work also allows the instructor to accurately assess the participation and contributions of each individual, and to assign appropriate grades. (4) Finally, the project introduces the students to electronic networking, and gives them a realistic opportunity to practice using it.

\section{REFERENCES}

Albrektson, J. R. (1995). Mentored online seminar: A model for graduate-level distance learning. Technological Horizons in Education, 23(3), 102-105.

MASON, R. (ED.) (1993). Computer conferencing: The last word. Victoria, BC: Beach Holme Publishers.

SLAVIN, R. E. (1990). Cooperative learning: Theory, research and practice. Englewood Cliffs, NJ: Prentice-Hall.

(Manuscript received November 13, 1995; accepted for publication January 3, 1996.) 